\title{
Allogeneic stem cell transplantation alone is unlikely to provide the HIV-1 cure
}

\author{
Nathan W Cummins ${ }^{1}$ \& Andrew D Badley*,1,2 \\ ${ }^{1}$ Division of Infectious Diseases, Mayo Clinic, Rochester, MN 55905, USA \\ ${ }^{2}$ Office of Translation to Practice, Mayo Clinic, Rochester, MN 55905, USA \\ *Author for correspondence: Tel.: +1 507284 1646; badley.andrew@mayo.edu
}

\section{' 6 It is natural, then, to question why the cure of 'Berlin patient' occurred in the first place, and whether this singular event could be recapitulated."}

First draft submitted: 15 January 2018; Accepted for publication: 8 February 2018; Published online: 19 April 2018

Keywords: allogeneic stem cell transplantation $\bullet$ HIV-1 cure

Despite the great success in the development of effective combination antiretroviral therapy, and the transformation of a once universally fatal infection into a manageable chronic condition, there remains no 'cure' for HIV-1 infection. This is remarkable, but not surprising. Remarkable because, despite the tens of millions of people historically and currently infected with HIV-1, to date there has been just one bona fide case of HIV-1 cure [1]. Not surprising though, due to the integration of the virus into the host genome, the resistance of infected cells to cell death [2] and the persistence and stability of the latent viral reservoir [3] which is unaffected by current antiretroviral medications.

It is natural, then, to question why the cure of 'Berlin patient' occurred in the first place, and whether this singular event could be recapitulated. That one cure occurred within the context of several rare events: myeloablative chemotherapy for a hematologic malignancy (acute myelogenous leukemia); two successive allogeneic stem cell transplantations from a donor homozygous for the protective CCR5 32 mutation rendering the allograft cells resistant to HIV-1 infection; and subsequent graft versus host disease (GVHD). Since intentionally repeating all three of these events in another patient would be virtually impossible and unnecessarily risky compared with suppressive antiretroviral therapy alone, it is reasonable then to ask if any one of these events might provide another HIV-1 cure.

Worldwide clinical experience treating malignancies in HIV-1 positive patients with a broad range of cytotoxic chemotherapeutic agents over several decades has failed to cure any other patient of HIV-1. However, newer antineoplastic agents are being repurposed in the search for a HIV-1 cure, including histone deacetylase inhibitors to reactivate latent HIV-1 and immune checkpoint inhibitors, among others [4]. We have recently shown that treatment of ex vivo CD4 T cells from HIV-1 positive patients with venetoclax, a BCL-2 specific inhibitor which is FDA-approved for the treatment of chronic lymphocytic leukemia, reduces HIV-1 reservoir size by sensitizing infected cells to HIV-induced apoptosis [5].

We, and others, have reported additional cases of allogeneic stem cell transplantation which were associated with reduced HIV-1 reservoir size, but ultimately without HIV-1 cure, suggesting that allogeneic transplantation is insufficient [6-8]. Notably, these cases involved donors with wild type CCR5. However, co-receptor tropism switch and viral escape of $C C R 5 \triangle 32$ selection in stem cell transplantation have been reported, suggesting modulation of CCR5 expression is also not sufficient for HIV-1 cure [9]. Furthermore, there is currently no reliable biomarker of HIV-1 reservoir size that is sensitive enough to adequately predict HIV-1 cure [10,11]. In fact, in the cases referenced above, multiple independent measures of HIV-1 were at or below the limit of detection, similar to the 'Berlin patient' [12] and yet viral rebound occurred.

Finally, our recent data [8] suggest that clinical GVHD may not have a graft versus HIV effect as previously hypothesized. In our reported patient, clinical GVHD was associated with increased expression of immune activation markers on a number of immune cell types, and occurred coincidentally with evidence of clonal expansion of latent 
HIV-1 sequences. Therefore, GVHD may induce homeostatic proliferation and persistence of cells containing latent HIV-1, rather than immune-mediated clearance of residual recipient-derived cells.

Therefore, it is unlikely that allogeneic stem cell transplantation alone could provide a method for HIV-1 cure, at least at a success rate and cost that would justify the significant attendant risks. However, it still remains an important area to investigate, as few if any other treatments have been shown to reduce the HIV-1 reservoir size to any degree. Important questions remain, including: effects of different conditioning regimens on pre-transplant reservoir size; effects of donor and recipient genetic mutations other than CCR5 on changes in reservoir size after transplant; the role of extra lymphoid tissue HIV-1 reservoirs in viral persistence and relapse; the potential role of prophylactic or therapeutic HIV-1 vaccination after transplantation to boost anti-HIV immunity in an otherwise naive immune system; and the role of immune activation from GVHD or opportunistic infections in promoting viral persistence.

The idea that a single 'magic bullet' intervention will result in an HIV-1 cure has largely been abandoned. However, careful and systematic observation of HIV-1 positive individuals who are undergoing allogeneic stem cell transplantation for another reason, such as a hematologic malignancy, may identify combinations of interventions that lead to substantial reductions in HIV-1 reservoir size, and thereby lead to new insights and novel targets for a potential future multimodality HIV-1 cure.

\section{Financial \& competing interests disclosure}

This work was supported by funding from the National Institutes of Allergy and Infectious Diseases R01 Al120698 and Al110173 and the Mayo Clinic Foundation. The authors have no other relevant affiliations or financial involvement with any organization or entity with a financial interest in or financial conflict with the subject matter or materials discussed in the manuscript apart from those disclosed.

No writing assistance was utilized in the production of this manuscript.

\section{Disclaimer}

The contents of this article are solely the responsibility of the authors and do not necessarily represent the official view of NIH or Mayo Clinic.

\section{References}

1. Hutter G, Nowak D, Mossner M et al. Long-term control of HIV by CCR5 Delta32/Delta32 stem-cell transplantation. N. Engl. J. Med. 360(7), 692-698 (2009).

2. Cummins NW, Badley AD. Anti-apoptotic mechanisms of HIV: lessons and novel approaches to curing HIV. Cell Mol. Life Sci. 70(18), 3355-3363 (2013).

3. Siliciano JM, Siliciano RF. The remarkable stability of the latent reservoir for HIV-1 in resting memory CD $4^{+} \mathrm{T}$ cells. J. Infect. Dis. 212(9), 1345-1347 (2015).

4. Rasmussen TA, Anderson JL, Wightman F, Lewin SR. Cancer therapies in HIV cure research. Curr. Opin. HIV AIDS 12(1), 96-104 (2017).

5. Cummins NW, Sainski AM, Dai H et al. Prime, shock, and kill: priming CD4 T cells from HIV patients with a BCL-2 antagonist before HIV reactivation reduces HIV reservoir size. J. Virol. 90(8), 4032-4048 (2016).

6. Henrich TJ, Hu Z, Li JZ et al. Long-term reduction in peripheral blood HIV type 1 reservoirs following reduced-intensity conditioning allogeneic stem cell transplantation. J. Infect. Dis. 207(11), 1694-1702 (2013).

7. Henrich TJ, Hanhauser E, Marty FM et al. Antiretroviral-free HIV-1 remission and viral rebound after allogeneic stem cell transplantation: report of 2 cases. Ann. Intern. Med. 161(5), 319-327 (2014).

8. Cummins NW, Rizza S, Litzow MR et al. Extensive virologic and immunologic characterization in an HIV-infected individual following allogeneic stem cell transplant and analytic cessation of antiretroviral therapy: a case study. PLoS Med. 14(11), e1002461 (2017).

9. Kordelas L, Verheyen J, Beelen DW et al. Shift of HIV tropism in stem-cell transplantation with CCR5 Delta32 mutation. N. Engl. J. Med. 371(9), 880-882 (2014).

10. Bruner KM, Hosmane NN, Siliciano RF. Towards an HIV-1 cure: measuring the latent reservoir. Trends Microbiol. 23(4), 192-203 (2015).

11. Massanella M, Richman DD. Measuring the latent reservoir in vivo. J. Clin. Invest. 126(2), 464-472 (2016).

12. Yukl SA, Boritz E, Busch M et al. Challenges in detecting HIV persistence during potentially curative interventions: a study of the Berlin patient. PLoS Pathog. 9(5), e1003347 (2013). 